\title{
Piomiositis tuberculosa en un paciente inmunosuprimido
}

\author{
Johanna Osorio ${ }^{1,2,3}$, Jackeline Barreto ${ }^{1,3}$, Jhonattan Benavides ${ }^{1,2}$, \\ Óscar López ${ }^{2}$, Ángela Cuenca ${ }^{3}$, Esperanza García ${ }^{4}$ \\ 1 Grupo de Investigación Infectocontrol, Hospital Universitario de Neiva, Neiva, Colombia \\ 2 Departamento de Medicina Interna, Facultad de Salud, Universidad Surcolombiana, Neiva, Colombia \\ 3 Unidad de Infectología, Hospital Universitario de Neiva, Neiva, Colombia \\ 4 Laboratorio Clínico y de Microbiología, Hospital Universitario de Neiva, Neiva, Colombia \\ Institución donde se realizó el trabajo: Hospital Universitario de Neiva, Neiva, Colombia \\ La piomiositis tuberculosa es una manifestación poco frecuente de la tuberculosis extrapulmonar que \\ se presenta más comúnmente en pacientes inmunosuprimidos, con manifestaciones clínicas similares \\ a las de la piomiositis de otras etiologías, pero a una edad más temprana. Los factores de riesgo \\ más usuales son la infección tuberculosa previa y la inmunosupresión farmacológica. El diagnóstico \\ depende de la sospecha clínica en una población expuesta, ya que en muchas ocasiones el aislamiento \\ microbiológico no es posible. La respuesta al tratamiento y el pronóstico son buenos. \\ El caso que se presenta es llamativo dada la rareza de esta manifestación de la tuberculosis y la lenta \\ mejoría con el tratamiento antituberculoso de primera línea en este paciente con infección por HIV y \\ recaída, a pesar de que el aislamiento microbiológico resultó sensible.
}

Palabras clave: tuberculosis/terapia, VIH, piomiositis.

doi: http://dx.doi.org/10.7705/biomedica.v36i3.2660

\section{Tuberculous pyomyositis in an immunosuppressed patient}

Tuberculous pyomyositis is a rare manifestation of extrapulmonary tuberculosis, most common in immunosuppressed patients, with clinical manifestations similar to pyomyositis of other etiologies, although with a lower age of presentation; notable risk factors include prior tuberculosis infection and pharmacological immunosuppression. Diagnosis depends on a high clinical suspicion of the infection in a susceptible population, given that microbiological isolation is often impossible. The response to treatment and prognosis are good.

The case presented here is noteworthy given the rarity of this manifestation of tuberculosis and the slow response to first-line TB management in an HIV patient, despite susceptible microbiological isolation.

Key words: tuberculosis/therapy, HIV, pyomyositis.

doi: http://dx.doi.org/10.7705/biomedica.v36i3.2660

La infección por Mycobacterium tuberculosis en los pacientes con virus de la inmunodeficiencia humana (HIV) tiene características y manifestaciones clínicas particulares que la diferencian de la que se presenta en pacientes inmunocompetentes, lo que explica la dificultad de su diagnóstico en esta población. Se estima que para el 2013, nueve millones de personas tenían la enfermedad, de las cuales $13 \%$ presentaba, además, la infección por HIV. En pacientes inmunocompetentes la forma pulmonar de la tuberculosis es la manifestación más frecuente, pero en uno de cada cinco casos se presenta en la forma extrapulmonar, aunque en los pacientes con infección concomitante puede llegar a más de $50 \%$ (1-3).

En Colombia, la tuberculosis y el HIV/sida son problemas de salud pública $y$, a pesar de ser enfermedades de notificación obligatoria, aún hay un importante subregistro, especialmente de las formas extrapulmonares de la tuberculosis, entre las que predominan la miliar, la pleural, la ganglionar, la meníngea y la osteoarticular $(4,5)$. Estas formas se caracterizan por la dificultad de su diagnóstico, y de la confirmación clínica y

\footnotetext{
Contribución de los autores:

Johanna Osorio: asesoría temática y metodológica

Jhonattan Benavides y Jackeline Barreto: revisión de la historia clínica y búsqueda de la literatura científica pertinente

Ángela Cuenca: recolección de datos y revisión de la historia clínica

Esperanza García: diagnóstico microbiológico e interpretación de los resultados

Todos los autores participaron en la escritura del manuscrito.
} 
bacteriológica, así como su presentación atípica en sitios inaccesibles. Se describe a continuación un caso de tuberculosis con compromiso musculoesquelético en un paciente con HIV/sida y tuberculosis pulmonar en tratamiento por recaída.

\section{Presentación del caso}

Se trata de un hombre de 31 años de edad procedente del área rural de Campoalegre, Huila, que consultó por un cuadro clínico de seis días de evolución consistente en fiebre de predominio nocturno, dolor abdominal de tipo cólico en mesogastrio irradiado al flanco derecho y la región lumbar, y abundantes deposiciones líquidas con moco un día antes de la hospitalización.

Al paciente se le había diagnosticado infección por HIV seis años antes, y no había recibido tratamiento antirretroviral en los últimos dos años. En el momento del diagnóstico de la infección por retrovirus, presentaba tuberculosis pulmonar, la cual recibió tratamiento de categoría I (48 dosis en la primera fase y 36 en la segunda); el cultivo de esputo de control resultó negativo. En los cuatro meses previos había sido hospitalizado en varias ocasiones por diarrea y síntomas respiratorios. Dos meses antes de la hospitalización se había confirmado la reaparición de la tuberculosis pulmonar mediante tinción de Ziehl-Neelsen en esputo positiva para bacilos ácido-alcohol resistentes, por lo cual se encontraba finalizando la primera fase del tratamiento de primera línea con rifampicina, isoniazida, pirazinamida y etambutol. En esa ocasión, en el estudio de sensibilidad de M. tuberculosis a los fármacos antituberculosos se reportó contaminación de la muestra. Un mes antes de la hospitalización, la carga viral del HIV fue de 319.860 copias $/ \mathrm{ml} \mathrm{y}$, el recuento de linfocitos T CD4+, de 41 células/ $\mu$ l.

En el examen de ingreso se detectaron adenomegalias cervicales y supraclaviculares, hepatomegalia palpable a $4 \mathrm{~cm}$ por debajo del reborde costal y dolor a la palpación en el flanco derecho con percusión ipsilateral positiva.

En los exámenes paraclínicos de ingreso se encontró: hemograma con 5.900 leucocitos por $\mathrm{mm}^{3}, 72,3 \%$ de neutrófilos, $12,8 \%$ de linfocitos,

\footnotetext{
Correspondencia:

Johanna Osorio, Carrera $55 \mathrm{~N}^{\circ} 11-49$, torre 1C, apartamento 207, Reserva de la Sierra, Neiva, Huila

Celular: (318) 3612729

osoriopinzon@gmail.com

Recibido: 03/02/15; aceptado: 02/12/15
}

$10,4 \%$ de monocitos, $1,6 \%$ de eosinófilos y $0,3 \%$ de basófilos. Otros valores fueron: hemoglobina, 10,4 $\mathrm{g} / \mathrm{dl}$; hematocrito, 31,9 \%; plaquetas, 106.000 por $\mathrm{mm}^{3}$; proteína $\mathrm{C}$ reactiva, $16 \mathrm{mg} / \mathrm{dl}$; sodio, 133,9 mEq/l; potasio, 3,57 mEq/L; calcio iónico, $1,152 \mathrm{mEq} / \mathrm{L}$, cloro, $99,5 \mathrm{mEq} / \mathrm{L}$; glucosa en suero, $105,1 \mathrm{mg} / \mathrm{dl}$; nitrógeno ureico, $10 \mathrm{mg} / \mathrm{dl}$; creatinina sérica, $0,55 \mathrm{mg} / \mathrm{dl}$; bilirrubina total, $0,41 \mathrm{mg} / \mathrm{dl}$; bilirrubina indirecta, $0,3 \mathrm{mg} / \mathrm{dl}$; transaminasa glutámico-oxaloacética (TGO), 19,1 UI/L; transaminasa glutámico-pirúvica (TGP), 55,7 UI/L; deshidrogenasa láctica, $179 \mathrm{UI} / \mathrm{L}$; y fosfatasa alcalina, 294,6 UI/L. El examen seriado de esputo con tinción de Ziehl-Neelsen fue negativo. En la radiografía de tórax se observaron infiltrados parahiliares y opacidad apical bilateral.

Durante la evolución, el paciente presentó disminución de la diarrea, pero el dolor abdominal persistió, por lo que se tomó una tomografía abdominal con contraste, en la que se observó heterogeneidad en la densidad del músculo psoas derecho con una colección líquida de 66 × 44 $\mathrm{mm}$ de diámetro y pequeñas colecciones satélite menores de $1 \mathrm{~cm}$ (figura 1).

Se inició el tratamiento con vancomicina y ampicilina más sulbactam por sospecha de piomiositis primaria del psoas. En la unidad de radiología intervencionista se hizo el drenaje percutáneo del absceso y se obtuvo un líquido de aspecto purulento que resultó negativo para gérmenes comunes con la coloración de Gram y la tinción de Ziehl-Neelsen, y en el cultivo. Al quinto día del drenaje se hizo un estudio con ecografía, en el cual se confirmó la desaparición de la colección, por lo que se retiró la sonda de drenaje, y se continuó con la administración de los antibióticos mencionados hasta completar tres semanas. El tratamiento antirretroviral se reinició al término de la primera fase del tratamiento antituberculoso y se prescribió profilaxis primaria con claritromicina para el complejo Mycobacterium avium debido a que el recuento de CD4 era menor de 50 células/ $\mathrm{mm}^{3}$, y con trimetropim más sulfametoxazol para Pneumocystis jirovecii, en concordancia con las recomendaciones internacionales para la prevención de infecciones oportunistas en pacientes con HIV (6).

En el día 21 del tratamiento antibiótico, el paciente presentó fiebre, por lo que se le practicó una tomografía de control que evidenció la persistencia de la colección en el psoas derecho. Se hizo un nuevo drenaje por vía percutánea y se reportaron 

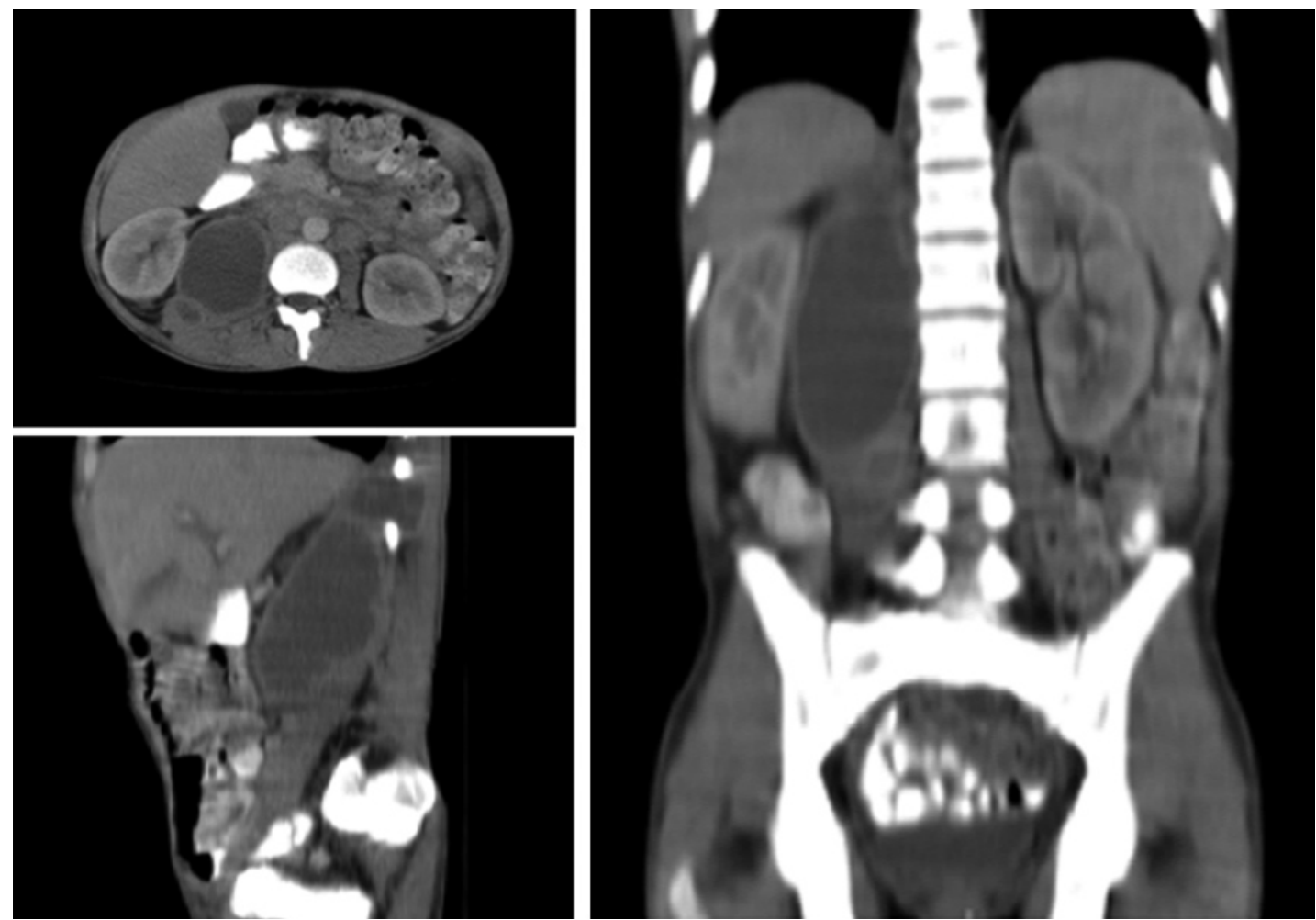

Figura 1. Tomografía abdominal con contraste que muestra heterogeneidad en la densidad del músculo psoas derecho. Imagen sugestiva de un absceso de 66 × $44 \mathrm{~mm}$ de diámetro, además de pequeñas colecciones satélite menores de $1 \mathrm{~cm}$

resultados negativos para gérmenes comunes con la coloración de Gram, la de Ziehl-Neelsen, con hidróxido de potasio al $10 \%$ y en los cultivos. Se decidió simplificar el esquema antibiótico con ceftaroline y se retiró el catéter al sexto día de drenaje al comprobar la desaparición de la colección.

En la tercera semana del nuevo esquema de tratamiento se le practicó al paciente una ecografía, la cual evidenció la reaparición de la colección en la misma localización, aunque de menor tamaño, por lo que se hizo un nuevo drenaje; esta vez el líquido se sembró en medio de cultivo Bactec Myco/F Lytic ${ }^{\circledR}$ y la botella se mantuvo en un equipo Bactec $9000^{\circledR}$ durante 42 días, según el protocolo. Al cabo de cuatro días, 13 horas y 37 minutos de incubación la muestra resultó positiva.

Según el protocolo, se prepararon láminas para la coloración de Gram, de Ziehl-Neelsen, de tinta china e hidróxido de potasio al 10\%, con lo cual se detectaron bacilos ácido-alcohol resistentes (figura 2); posteriormente, se sembraron en agar sangre, agar MacConkey y medio Ogawa-Kudoh. A las tres semanas de incubación se registró el crecimiento de colonias secas, rugosas, con una leve coloración marfil en toda la superficie del

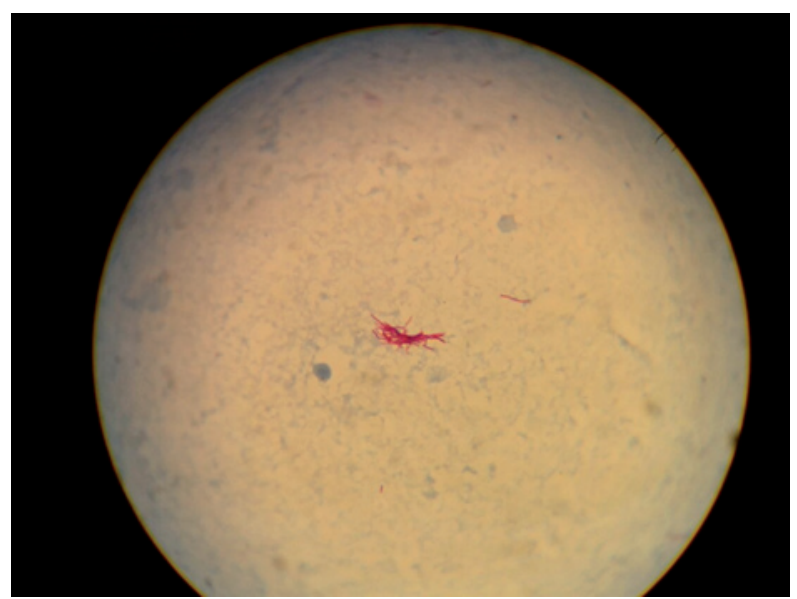

Figura 2. Tinción de Ziehl-Neelsen del líquido drenado del absceso en el psoas, la cual evidenció bacilos ácido-alcohol resistentes, $100 \mathrm{X}$

medio, las cuales se enviaron al laboratorio de la Secretaría Departamental de Salud para su identificación y para las pruebas de sensibilidad. El cultivo fue positivo para $M$. tuberculosis en la prueba Bactec MGIT $960^{\circledR}$ con sensibilidad confirmada a la isoniacida y la rifampicina, según el reporte del Instituto Nacional de Salud. 
Posteriormente, se confirmó por ecografía la desaparición de la colección retroperitoneal; la fiebre, el dolor y los demás síntomas también remitieron. El paciente fue dado de alta dada su evolución favorable, y se consideró extender el tratamiento antituberculoso a nueve meses.

\section{Discusión}

La tuberculosis es un importante problema de salud pública y cuando se presenta concomitantemente con infección por HIV, son más frecuentes las formas extrapulmonares. El surgimiento del HIV ha tenido un notorio impacto en la historia natural y la epidemiología de la tuberculosis en el ámbito global. De los nueve millones de casos nuevos de la enfermedad en el 2013, la Organización Mundial de la Salud estimó que cerca de 1,0 a 1,2 millones (11 a $14 \%$ ) correspondían a personas con HIV $(1,7)$. En Colombia, se presentan alrededor de 12.000 nuevos casos de tuberculosis al año; para la semana epidemiológica 44 de 2014 se habían reportado 10.639 casos, de los cuales 8.547 eran pulmonares, 1.711 de formas extrapulmonares y en 381 hubo compromiso meníngeo. De todos los casos, 0,6 \% correspondió a fracasos del tratamiento.

La localización extrapulmonar más frecuente fue la pleural (33,3\%), seguida de la meníngea $(18,4 \%)$, pero no se reportaron casos de localización muscular durante ese periodo. La asociación de tuberculosis e infección por HIV fue de $31 \%$ y. en los casos extrapulmonares llegó a $44,1 \%$. Según la notificación oficial, el departamento del Huila presentaba una tasa de incidencia de tuberculosis pulmonar de 22,1 casos por cada 100.000 habitantes y, de 2,6 por 100.000 , de formas extrapulmonares (5).

La piomiositis del psoas es una entidad poco frecuente, la cual se clasifica, según su origen, en primaria (30\% de los casos) o secundaria a procesos inflamatorios renales, osteoarticulares o gastrointestinales. En el caso de la enfermedad primaria asociada a infección por HIV, el microorganismo más frecuentemente aislado es $M$. tuberculosis, seguido de Staphlylococcus aureus resistente a la meticilina adquirido en la comunidad. La diseminación hematógena es la principal fuente de infección, lo cual se ha demostrado en modelos experimentales después de un traumatismo muscular (8). En una serie de casos de piomiositis (9), el compromiso del tronco se encontró en 27,2\% de los casos, y se logró identificar el microorganismo en $74 \%$ de ellos, y $S$. aureus fue el agente más frecuentemente aislado $(88,8 \%)$. Vale la pena señalar que los cultivos negativos llegaron a representar el $8 \%$, lo que podría relacionarse con gérmenes de crecimiento exigente. En dicho reporte de casos no hubo aislamientos de micobacterias, probablemente debido a la exclusión de pacientes con enfermedades concomitantes como la infección por HIV, y a que la serie incluía solamente pacientes que habían respondido a la oxacilina como único antibiótico.

En otra serie de 124 pacientes (10) con diagnóstico confirmado de absceso en el psoas, en la cual se incluyeron pacientes con infección por HIV, la edad media de presentación para este grupo fue menor comparada con la de los pacientes sin HIV (35,5 Vs. 60,5 años). Los microorganismos más frecuentemente aislados fueron $M$. tuberculosis (50 \%), seguido por S. aureus y Escherichia coli. El $26,7 \%$ de los pacientes con absceso tuberculoso tenía infección con HIV, lo que constituyó un factor de riesgo significativo frente a aquellos sin HIV $(p=0,007)$. La fuente primaria de absceso tuberculoso fue menos frecuente que la secundaria (14\% Vs. $86 \%$ ) y el origen óseo fue el más común. Hubo, además, asociación con el síndrome de reconstitución inmunitaria por desenmascaramiento después de iniciado el tratamiento antirretroviral combinado (11), lo que se descartó en el paciente de este caso, ya que no estaba recibiendo antirretrovirales en el momento del diagnóstico de piomiositis.

Pese al elevado número de casos asociados a M. tuberculosis, en la mayoría no fue posible la confirmación microbiológica y muchas veces se hizo el diagnóstico con base en los criterios clínicos y epidemiológicos; en ocasiones, la fuerte sospecha clínica y la reacción al tratamiento antituberculoso sirvieron de soporte para el diagnóstico. El rendimiento de los cultivos de absceso fue similar a lo reportado en los casos no tuberculosos, con independencia del germen causal (46 a $68 \%$ ), pero no fue posible el aislamiento en sangre de las micobacterias, en tanto que los hemocultivos permitieron hacer el diagnóstico en algunos casos relacionados con bacterias comunes.

En pacientes sin HIV, hasta $2 \%$ de las tuberculosis asociadas al sistema osteomuscular corresponden a piomiositis (12). Otras condiciones que se asocian a abscesos musculares tuberculosos son el uso crónico de corticoides, la medicación inmunosupresora (metrotexato-azatioprina), el tratamiento con infliximab, las afecciones crónicas 
(fibrosis pulmonar, sarcoidosis, dermatomiositis, polimiositis, artritis reumatoidea y lupus eritematoso sistémico) y el trasplante renal (13-16). Existen reportes en pacientes inmunocompetentes $(17,18)$ que ratifican que no siempre la manifestación es insidiosa y larvada, y se han observado casos de sepsis y muerte a causa de la infección, pero también, de la toxicidad de los medicamentos. En la mayoría de los casos reportados la respuesta terapéutica ha sido buena, independientemente de la infección concomitante.

Este caso de piomiositis tuberculosa con infección por HIV ya en estadio de sida es poco frecuente, y se manifestó a una edad similar a la reportada en otros estudios. En muchos de los casos publicados, el único criterio utilizado para el diagnóstico de la piomiositis tuberculosa fue la alta sospecha clínica y la respuesta al tratamiento. Este caso llama la atención porque el paciente presentó una mejoría lenta con los fármacos antituberculosos primera línea, y el cultivo para micobacterias del material drenado del absceso fue positivo al culminar la primera fase del tratamiento. Este comportamiento se ha observado en individuos con avanzado estado de inmunosupresión, bajos conteos de CD4 y desnutrición $(19,20)$, a lo que, en este caso puntual, se sumaron las dificultades para absorber los medicamentos debido a la enfermedad diarreica crónica. El paciente tenía factores de riesgo para la multirresistencia (HIV y tratamiento antituberculoso previo) (21-23), a pesar de que durante su hospitalización se confirmó la presencia de infección por $M$. tuberculosis con sensibilidad a la medicación de primera línea (rifampicina, isoniacida).

En general, los factores que se deben tener en cuenta ante la sospecha de fracaso en el tratamiento, son el cumplimiento cabal de este, la interrupción de los medicamentos por efectos adversos, las interacciones farmacológicas, la malabsorción, la resistencia o los errores en su tipificación y evaluación, así como los defectos en la elaboración de los medicamentos (24).

\section{Conclusión}

La piomiositis tuberculosa es una presentación clínica inusual de la tuberculosis extrapulmonar de muy baja frecuencia en pacientes inmunocompetentes e, incluso, en aquellos con HIV/ sida. El caso clínico expuesto es importante, pues se trata de una presentación típica de una condición poco frecuente, de difícil diagnóstico etiológico y con una respuesta que no fue óptima a los medicamentos de primera línea, a pesar de tener sensibilidad confirmada. En los pacientes con factores de riesgo, se requiere de una fuerte sospecha clínica, especialmente en aquellos con diagnóstico previo de tuberculosis que viven en áreas endémicas.

\section{Conflicto de intereses}

Los autores declaramos no tener ningún tipo de conflicto de intereses con respecto al manuscrito.

\section{Financiación}

Los autores declaramos que no tuvimos fuente de financiación para este artículo.

\section{Referencias}

1. World Health Organization. Global tuberculosis report 2014. WHO/HTM/TB/2014.08. Fecha de consulta: 1ำ de mayo 2015. Disponible en: www.who.int/tb/publications/ global_report/gtbr14_main_text.pdf.

2. Cortés JA, Hidalgo P, Rey DL, Parra GA, Gutiérrez IF. Tuberculosis en pacientes con infección por VIH en el Hospital Universitario de San Ignacio 2002-2006. Infectio. 2007;11:16-22.

3. Lozada C, Lemus LF, Guevara J, Velasco M, Chan L, Leal VC. Tuberculosis extrapulmonar, reporte de caso. Revista Colombiana Salud Libre. 2011;10:80-89.

4. Díaz M, Muñoz S, García L. Tuberculosis en el Hospital Universitario San José, Popayán, 1998-2000. Biomédica. 2004;24(Supl.1):92-101. http://dx.doi.org/10.7705/biomedica. v24iSupp+1.1308

5. Instituto Nacional de Salud. Boletín Epidemiológico Semanal № 44 de 2014 (26 octubre a 1 noviembre de 2014). Bogotá: INS; 2014. p. 28-9. Fecha de consulta: 1 de mayo de 2015. Disponible en: http://www.ins.gov.co/ boletin-epidemiologico/Boletn\%20Epidemiolgico/2014\%20 Boletin\%20epidemiologico\%20semana\%2044.pdf.

6. Panel on Opportunistic Infections in HIV-Infected Adults and Adolescents. Guidelines for prevention and treatment of opportunistic infections in HIV-infected adults and adolescents: Recommendations from the Centers for Disease Control and Prevention, the National Institutes of Health, and the HIV Medicine Association of the Infectious Diseases Society of America. 2015. Fecha de consulta: 1으 de mayo de 2015. Disponible en: https://aidsinfo.nih.gov/ contentfiles/lvguidelines/Adult_OI.pdf.

7. Arenas NE, Ramírez N, González G, Rubertone S, García AM, Gómez JE, et al. Estado de la coinfección tuberculosis/ virus de la inmunodeficiencia humana en el municipio de Armenia (Colombia): experiencia de 10 años. Infectio. 2012;16:140-7.

8. Shields D, Robinson P, Crowley TP. Iliopsoas abscess-a review and update on the literature. Int J Surg. 2012;10: 4669. http://dx.doi.org/10.1016/j.ijsu.2012.08.016.

9. Campbell S, Iglesias A. Piomiositis. Informe de 132 pacientes. Biomédica. 1994;14:105-16. http://dx.doi.org/10. 7705/biomedica.v14i2.2093 
10. Navarro-López V, Ramos JM, Meseguer V, PérezArellano JL, Serrano R, García-Ordóñez MA, et al. Microbiology and outcome of iliopsoas abscess in 124 patients. Medicine (Baltimore). 2009;88:120-30. http://dx. doi/10.1097/MD.0b013e31819d2748

11. Chen WL, Lin YF, Tsai WC, Tsao YT. Unveiling tuberculous pyomyositis: An emerging role of immune reconstitution inflammatory syndrome. Am J Emerg Med. 2009;27:251. e1-2. http://dx.doi.org/10.1016/j.ajem.2008.05.030.

12. Muangchan C, Nilganuwong S. The study of clinical manifestation of osteoarticular tuberculosis in Siriraj Hospital, Thailand. J Med Assoc Thai. 2009;92(Suppl.2):S101-9.

13. Krishnasamy V, Joseph M. Tuberculous pyomyositis: A rare but serious diagnosis. Case Rep Med. 2013;2013:126952. http://dx.doi.org/10.1155/2013/126952

14. Simopoulou T, Varna A, Dailiana Z, Katsiari C, Alexiou I, Basdekis G, et al. Tuberculous pyomyositis: A re-emerging entity of many faces. Clin Rheumatol. 2014:1-6. http://dx. doi/10.1007/s10067-014-2564-8

15. Mootsikapun P, Mahakkanukrauh A, Suwannaroj S, Nanagara R. Tuberculous pyomyositis. J Med Assoc Thai. 2003;86:477-81.

16. Indudhara R, Singh SK, Minz M, Yadav RV, Chugh KS. Tuberculous pyomyositis in a renal transplant recipient. Tuber Lung Dis. 1992;73:239-41.

17. Modi MA, Mate AD, Nasta AM, Gvalan AK. Primary tuberculous pyomyositis of quadriceps femoris in an immunocompetent individual. Case Rep Infect Dis. 2013; 2013:723879. http://dx.doi/10.1155/2013/723879
18. Sen RK, Tripathy SK, Deivaraju C, Das A. Isolated focal pyomyositis of teres minor: An unusual presentation of tuberculosis. 2011. Acta Orthop Traumatol Turc. 2011;45: 276-9. http://dx.doi/10.3944/AOTT.2011.2447

19. Schwander SK, Dietrich M, Mugyenyi P, Kityo C, Okwera A, Johnson $\mathrm{J}$, et al. Clinical course of human immunodeficiency virus type 1 associated pulmonary tuberculosis during short-course antituberculosis therapy. East Afr Med J. 1997;74:543-8.

20. Johnson JL, Okwera A, Vjecha MJ, Byekwaso F, Nakibali J, Nyole S, et al. Risk factors for relapse in human immunodeficiency virus type 1 infected adults with pulmonary tuberculosis. Int J Tuberc Lung Dis. 1997; $1: 446-53$

21. Melzer M, Gupta N, Petersen I, Cook S, Hall B. Previous treatment in predicting drug-resistant tuberculosis in an area bordering East London, UK. Int J Infect Dis. 2010; 14:717-22. http://dx.doi/10.1016/j.ijid.2010.02.2247

22. Daniel O, Osman E. Prevalence and risk factors associated with drug resistant TB in South West, Nigeria. Asian Pac J Trop Med. 2011;4:148-51. http://dx.doi/10.1016/S19957645(11)60057-6

23. Caminero JA. Multidrug-resistant tuberculosis: Epidemiology, risk factors and case finding. Int $\mathrm{J}$ Tuberc Lung Dis. 2010;14:382-90.

24. Bonomo RA, Graham R, Makley JT, Petersilge CA. Tuberculous pyomyositis: An unusual presentation of disseminated Mycobacterium tuberculosis infection. Clin Infect Dis. 1995;20:1576-7. http://dx.doi/10.1093/clinids/20. 6.1576 